157 PATIENT SATISFACTION AND SAFETY OF SEDATION USING PERINEURIAL DEXMEDETOMIDINE IN AXILLARY BRACHIAL PLEXUS BLOCK FOR WRIST SURGERY

${ }^{1}$ RP Ročāns*, ${ }^{2}$ M Andruškevičs, ${ }^{1}$ A Ozolina, ${ }^{1}$ B Mamaja. 'Riga Stradins University, Riga, Latvia; ${ }^{2}$ University of Latvia, Rīga, Latvia

10.1136/rapm-2021-ESRA.157

Background and Aims Dexmedetomidine is known to prolong the duration of regional block while its sedative effect when administered perineurally is unknown.[1] We aim to evaluate the effect of perineural Dexmedetomidine on the systemic sedation in patients after axillary brachial plexus block (ABPB).

Methods This prospective randomized control trial includes 78 patients undergoing wrist surgery receiving ABPB. The study was conducted with the approval of Ethics Committee of Riga Stradins University. Patients were randomized into two groups - control (CG) $(\mathrm{N}=39)$ and study group (SG) $(\mathrm{N}=39)$. Both groups received $\mathrm{ABPB}$ with a standard dose of local anaesthetics. The study group also received $100 \mathrm{mcg}$ of dexmedetomidine perineurally. Depth of sedation during surgery was evaluated using electroencephalography with Narcontrend Index (NI) and Ramsay Sedation Scale (RSS). Patient satisfaction with sedation was evaluated using a postoperative survey.

Results During 10 to 60 minutes after ABPB the median RSS was 4 and median NI was between 54 to 69 in SG. Median RSS decreased to 2 and median NI increased to 90 in 90 minutes after ABPB in SG. Patients in the CG had the median RSS of 2 and Narcotrend Index of 97 all throughout surgery. $(p=0.001)$ No significant differences in hemodynamic or respiratory parameters were found between the groups. Patients in the SG expressed satisfaction with quality of sedation and $88.4 \%$ noted that the sensation was similar to ordinary sleep.

Conclusions Perineural administration of Dexmedetomidine provides systemic sedation with no effect on hemodynamic or respiratory stability and yields a high level of patient satisfaction.

\section{TIMING OF FASCIA ILIACA COMPARTMENT BLOCK (FICB) FOR NECK OF FEMUR (NOF) FRACTURE IN THE ACCIDENT AND EMERGENCY DEPARTMENT (AED) AND THE OPERATING THEATRE}

E Benison*. Liverpool Hospitals NHS Foundation Trust, Liverpool, UK

\subsection{6/rapm-2021-ESRA.158}

Background and Aims FICB is recommended for NOF fracture in the AED to improve analgesia ${ }^{1}$ and as an adjuvant to anaesthesia during surgery ${ }^{2}$. Surgical repair of the fracture should be performed on the day of, or the day after, admis$\operatorname{sion}^{3}$. We evaluated the provision of FICB in AED and its potential to cause local anaesthetic (LA) toxicity when followed by a further block at time of surgery.

Methods All patients admitted to our institution with NOF fracture during October 2019 were retrospectively screened for FICBs. We recorded the time of: admission, FICB in AED, surgery, FICB in theatre and LA dose. Data was crossreferenced and validated against National Hip Fracture Database (NHFD) tables. Toxicity thresholds were taken from the

Abstract 158 Table 1
\begin{tabular}{|l|l|l|}
\hline Time (hh:mm) from & admission to surgery & block in AED to surgery \\
\hline Average & $34: 08$ & $26: 32$ \\
\hline Minimum & $08: 28$ & $06: 00$ \\
\hline Maximum & $140: 06$ & $57: 01$ \\
\hline
\end{tabular}

British National Formulary and medicines.org.uk websites. Ethical approval was granted.

Results 36 patients were identified. 14(39\%) received a FICB in AED, 6(16\%) a peripheral nerve block in theatre and $3(8 \%)$ received both. On average, each block delivered $100 \mathrm{mg}$ of L-bupivocaine. Table-1 details times from admission to surgery and from block in AED to surgery. The maximum recommended doses of L-bupivocaine in 24 hours and post-operative analgesia are respectively $400 \mathrm{mg}$ and $18.75 \mathrm{mg} / \mathrm{h}$.

Conclusions Pain promotes delirium ${ }^{2}$ and worsens outcomes hence effective pain relief between admission and surgery is key to NOF fracture treatment. Our data matched the NHFD tables and highlighted the potential for LA toxicity in patients receiving a FICB in AED and same-day-of-admission surgery.

\section{EFFECT OF THE ADDICTION OF DEXMEDETOMIDINE IN REDUCING THE EFFICIENT DOSE OF BUPIVACAINE IN ULTRASOUND GUIDED SUPRACLAVICULAR BLOCK}

M Elhamdi*, C Ghouibi, M Boussofara. Université de Tunis El Manar, Tunis, Tunisia

\subsection{6/rapm-2021-ESRA. 159}

Background and Aims This study was conducted to determine the role of dexmedetomidine (DEX) as an adjuvant in reducing the efficient dose of bupivacaine in supraclavicular block.

Methods A prospective study, including 40 patients ASA I and ASA II undergoing upper limb and hand surgery, who were randomly divided into 2 groups; group D received the fixed dose of $100 \mu \mathrm{g}$ of dexmedetomidine $(1 \mathrm{ml})$ added to a predefined dose of bupivacaine $0.25 \%$; Group $S$ received bupivacaine $0.25 \%$ with $1 \mathrm{ml}$ of normal saline (NS).Starting with the dose of $30 \mathrm{ml}$ of bupivacaine $0.25 \%$. The dose was reduced by $3 \mathrm{ml}$ every time the block was a success. the onset time of the block at $30 \mathrm{~min}$ at most defined as the success criteria. The minimal dose required to have a sensory and a motor block in each group was compared. Onset time and duration of sensory and motor blockage, as well as side effects were also studied.

Results The addition of $100 \mu \mathrm{g}$ of DEX did not reduce the minimum efficient dose of bupivacaine. Tis dose was $17 \mathrm{ml}$ in GD versus $18 \mathrm{ml}$ in GS, to have $50 \%$ of successful supraclavicular bloc [ED50].

We noted a prolonged blockage duration without a reduction in the onset time in GD, with no statistical difference between the two groups.

We noted complications in the GD only, 2 cases of bradycardia, 2 of Claude Bernard Horner and one case of hypotension. But no statistical difference between the two groups. Conclusions To have $50 \%$ of successful supraclavicular bloc [ED50]. 